\title{
Capsule Commentary on Ashburner et al., Electronic Physician Notifications to Improve Guideline-Based Anticoagulation in Atrial Fibrillation: a Randomized Controlled Trial
}

\author{
loannis Mastoris, $\mathrm{MD}^{7}$ and Alexander G. Mathioudakis, MD, MRCP2 \\ 'Division of Cardiovascular Diseases, University of Kansas Medical Center, The University of Kansas Health System, Kansas City, KS, USA; ${ }^{2}$ Division of \\ Infection, Immunity and Respiratory Medicine, The University of Manchester, Manchester, UK.
}

J Gen Intern Med 33(12):2190

DOI: $10.1007 / \mathrm{s} 11606-018-4678-1$

(c) The Author(s) 2018

$\mathrm{W}$ hile oral anticoagulation (OAC) can mitigate the fivefold increase of ischemic stroke risk that is associated with atrial fibrillation (AF), this is at the expense of an increased risk of bleeding that often discourages clinicians and patients from prescribing OAC. Different perceptions of ostensible risk for either stroke or bleeding risk between primary care physicians and cardiologists lead to different therapeutic strategies and outcomes. Patients seen by cardiologists are $40 \%$ more likely to be prescribed $\mathrm{OAC}$ for $\mathrm{AF}$, resulting in $40 \%$ lower risk of stroke, without a significant increase in their bleeding risk. ${ }^{1}$ Similarly, a TREAT-AF substudy confirms that cardiology care in AF is associated with reduction in both stroke risk and mortality, largely due to early prescription of OAC after diagnosis. ${ }^{2}$ Since most patients with $\mathrm{AF}$ are managed in primary care and as shown, are likely to receive suboptimal management, it is crucial to implement change and to tackle the significant healthcare inequalities between patients seen by a specialist or a primary care physician.

In this issue of the Journal, Ashburner et al. evaluated the impact of an electronic alert tool in increasing the prescription rate of $\mathrm{OAC}$ in patients with $\mathrm{AF}$ seen in primary care setting. ${ }^{3}$ While this study did not show any evidence that electronic alerts would be effective towards that direction, it illustrates the challenges of clinical decision making in starting OAC. It sheds light on the reasons that prompted primary care physicians to avoid OAC, which included paroxysmal AF, perceived high bleeding risk, fall risk, and patient decision.

These concerning findings reveal a significant knowledge gap among primary care physicians, who believe their decisions not to use $\mathrm{OAC}$ are appropriate. As a result, patients also receive incorrect information that limit their ability to make an informed decision. A previous systematic review evaluating

Published online October 17, 2018 patient values and preferences in decision making for antithrombotic therapy revealed that well-informed patients place a higher disutility on stroke than bleeding or treatment burden and this choice must be respected by clinicians. ${ }^{4}$ Welldesigned educational interventions targeted to primary care physicians and patients are required to address this issue.

Corresponding Author: Ioannis Mastoris, MD; Division of Cardiovascular Diseases, University of Kansas Medical Center, The University of Kansas Health System, Kansas City, KS, USA (e-mail: imastoris@kumc.edu).

\section{Compliance with Ethical Standards:}

Conflict of Interest: The authors declare that they do not have a conflict of interest.

Open Access This article is distributed under the terms of the Creative Commons Attribution 4.0 International License (http:// creativecommons.org/licenses/by/4.0/), which permits unrestricted use, distribution, and reproduction in any medium, provided you give appropriate credit to the original author(s) and the source, provide a link to the Creative Commons license, and indicate if changes were made.

\section{REFERENCES}

1. O'Neal WT, Sandesara PB, Claxton JS, MacLehose RF, Chen LY, Bengtson LGS, et al. Provider specialty, anticoagulation prescription patterns, and stroke risk in atrial fibrillation. J Am Heart Assoc. 2018;7(6).

2. Perino AC, Fan J, Schmitt SK, Askari M, Kaiser DW, Deshmukh A, et al. Treating specialty and outcomes in newly diagnosed atrial fibrillation: from the TREAT-AF study. 2017;70(1):78-86.

3. Ashburner JM, Atlas SJ, Khurshid S, Weng LC, Hulme OL, Chang Y, Singer DE, Ellinor PT, Lubitz SA. Electronic Physician Notifications to Improve Guideline-Based Anticoagulation in Atrial Fibrillation: a Randomized Controlled Trial. J Gen Intern Med; doi: https://doi.org/10.1007/ s11606-018-4612-6.

4. MacLean S, Mulla S, Akl EA, Jankowski M, Vandvik PO, Ebrahim S, McLeod S, Bhatnagar N, Guyatt GG. Patient Values and Preferences in Decision Making for Antithrombotic Therapy: a systematic review: Antithrombotic Therapy and Prevention of Thrombosis, 9th ed: American College of Chest Physicians Evidence-Based Clinical Practice Guidelines. Chest. 2012;141(2 Suppl):e1S-e23S. doi: https://doi.org/10.1378/chest. 11-2290. 